\title{
A STUDY OF THE BLOOD PYRUVIC ACID LEVEL PATIENTS WITH CONGESTIVE HEART FAILURE
}

\author{
BY \\ J. KLEEBERG AND S. GITELSON \\ From the Medical Department of "A" Rothschild-Hadassah-University Hospital, Jerusalem
}

(RECEIVED FOR PUblication MAY 12, 1953)

The studies of the biochemical changes in heart failure are as interesting and important as those in the field of haemodynamics. The metabolic changes are not only the results of disturbed metabolism in circulatory or cardiac failure but in themselves may become influencing factors in failure.

Among the products of metabolism, pyruvic acid is of great importance because it is an intermediary in carbohydrate, as well as in protein and in fat metabolism. Since newer methods for its estimation have been introduced by Lu (1939) and by Friedemann and Haugen (1943), several authors have studied the role of pyruvic acid in heart diseases (Altschule and Rosenfeld, 1947 ; Barenghi, Scarlotti, and Scafidi, 1941 ; Lasch, 1952 ; Torre, Teece, and Baldantoni, 1950 ; Yanof, 1942), and a survey of the changes in pyruvic acid levels in other diseases and in relation to therapy has been published by Markees (1951a).

The purpose of this investigation was to study the level of blood pyruvic acid in patients with congestive heart failure and to ascertain: (1) whether the degree of severity of failure was reflected in changes of blood pyruvic acid values ; (2) whether any relationship existed between liver size, liver function, and blood pyruvic acid; (3) whether therapy would influence the blood pyruvic acid level.

\section{Clinical Data}

Ten healthy subjects fasting and at rest were chosen to obtain normal values of blood pyruvic acid. Forty patients, 20 men and 20 women, in various stages of congestive heart failure were also selected. The cardiac cases, classified according to aetiology, had arteriosclerotic or hypertensive cardiac and vascular diseases ( 20 cases); rheumatic heart disease (15 cases) ; chronic cor pulmonale (five cases).

Classified according to clinical severity, five patients had on admission no apparent signs of decompensation; 17 patients suffered from mil|户 or moderate failure; 14 were in severe failure and four were in very severe failure.

The patients were on a sodium-free diet. Mos of them had an intake of 1,500 to 2,000 calorieg and in addition received $50 \mathrm{mg}$. vitamin $B_{1}$. Treatment consisted of digitalis, cardophyllin, mercuriah diuretics, and dicoumarol adapted to the needs of each patient.

The effect of co-carboxylase on the blooe pyruvic acid level was studied in 13 patients. These patients were either given one single injection, oळ consecutive daily injections of 50 or $100 \mathrm{mg}$ intravenously.

\section{Methods}

For the determination of the blood pyruvic aci level Lu's hydrazin method as modified by Friede mann and Haugen (1943) was used, and Markees (1951b) technique of boiling the trichloracetic aciæ filtrate for five minutes to destroy aceto-acetiç. acid. Blood was always taken in the morning while the patient was fasting and in bed. In 10 patients a second estimation was done one or two hours after the first sample, with the patient stif fasting. In half of the cases further serial pyruvie. acid samples were taken, as many as 30 in ons patient. Blood sugar, cholesterol fractions, serum albumin and globulin, cephalin-flocculation, thy mol turbidity, and the Weltmann tests were als $0^{\circ}$ determined.

\section{Results}

The results are illustrated by Figs. 1 and 2 anẹ Table I.

The blood pyruvic acid values in 10 health $\bar{\Phi}$ individuals varied between 0.53 and $1.08 \mathrm{mg} . \%$.

Cardiac patients with no heart failure or with mild failure had normal pyruvic acid values Eighty per cent. of the values in moderate failure and nearly half the values in severe failure, nob complicated by acute events, were also norma? 


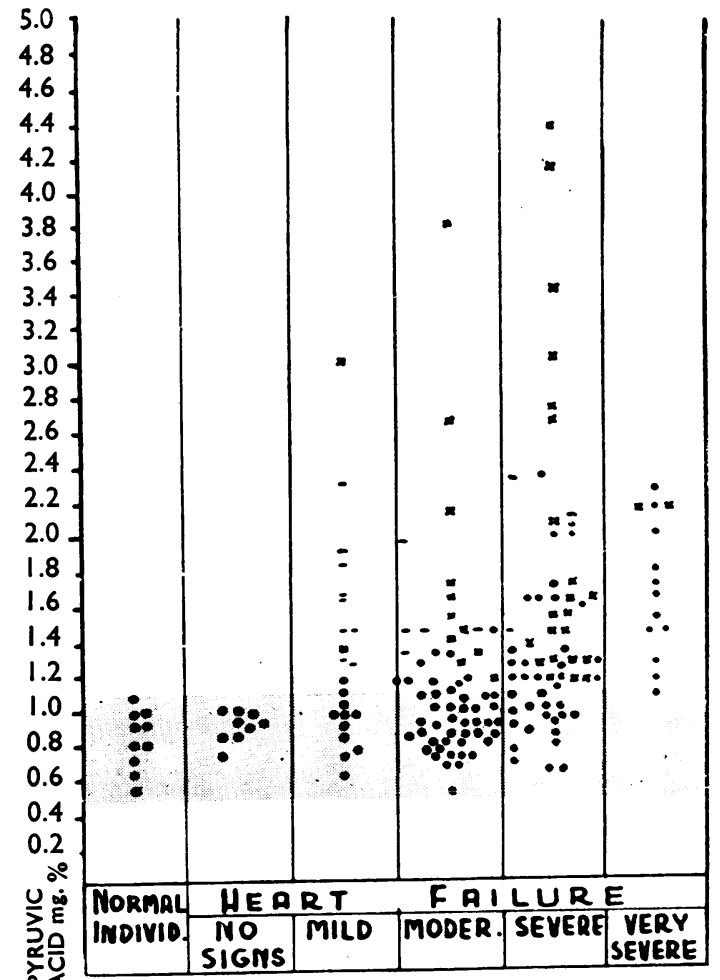

FIG. 1.-Distribution of blood pyruvic acid values in 40 cardiac patients and in 10 normal individuals. Crosses and dashes indicate values during or following severe complications. Shaded area indicates the range of normal values.

The remainder were slightly to moderately elevated (up to $2.3 \mathrm{mg} . \%$ ), as were all the values in the very severe cases. Especially high pyruvic acid levels (up to $4.4 \mathrm{mg}$.\%) were noted during and following severe complications, such as acute myocardial infarction, pulmonary oedema, hypertensive encephalopathy (crosses in Fig. 1 and Table I). Such hyperpyruvicaemia during acute episodes was found even in patients with mild failure (see Fig. 2). The pyruvic acid level showed a tendency to drop with clinical improvement, and to rise with the increase of the failure (see Table I).

There was no clear correlation between elevation of the blood pyruvic acid level and the extent of the liver damage, as indicated by the abovementioned tests.

High pyruvic acid levels were associated with elevated blood sugar values in over half the instances. On the other hand, high blood sugar values (in the non-diabetic patients) almost always coincided with an increase in pyruvic acid concentration in the blood.

Cases No. 24 and 28 will be described in more detail.

\section{Case Reports}

Case 24.-A. Z., a man aged 52, suffered from arterio-sclerotic heart and vascular disease, and recurrent myocardial infarction.

He was admitted to the hospital on December 5, 1951, four days after a recent infarction. He had moderate heart failure, and a slightly enlarged liver. Liver function tests were normal except for an increased urine urobilinogen excretion. Blood pyruvic acid values were high. There was rapid clinical improvement and a drop in the blood pyruvic acid level, remaining low during the following week. Subsequently there was a slow but steady rise in the blood pyruvic acid in spite of the patient feeling well, but suddenly the blood pyruvic acid level rose accompanied by an elevation in blood sugar after occasional anginal pains.

On January 10, 1952, the patient experienced sudden, transient shock with signs of fresh coronary infarction. The blood pyruvic acid level was very high accompanied once more by a rise in the blood sugar and the disappearance of blood eosinophils the following day. The heart failure increased in severity with further enlargement of the liver and scleral jaundice. During the next few days there was a temporary clinical improvement with a slight drop in the blood pyruvic acid and sugar levels. The patient died after a further acute event, probably a fresh

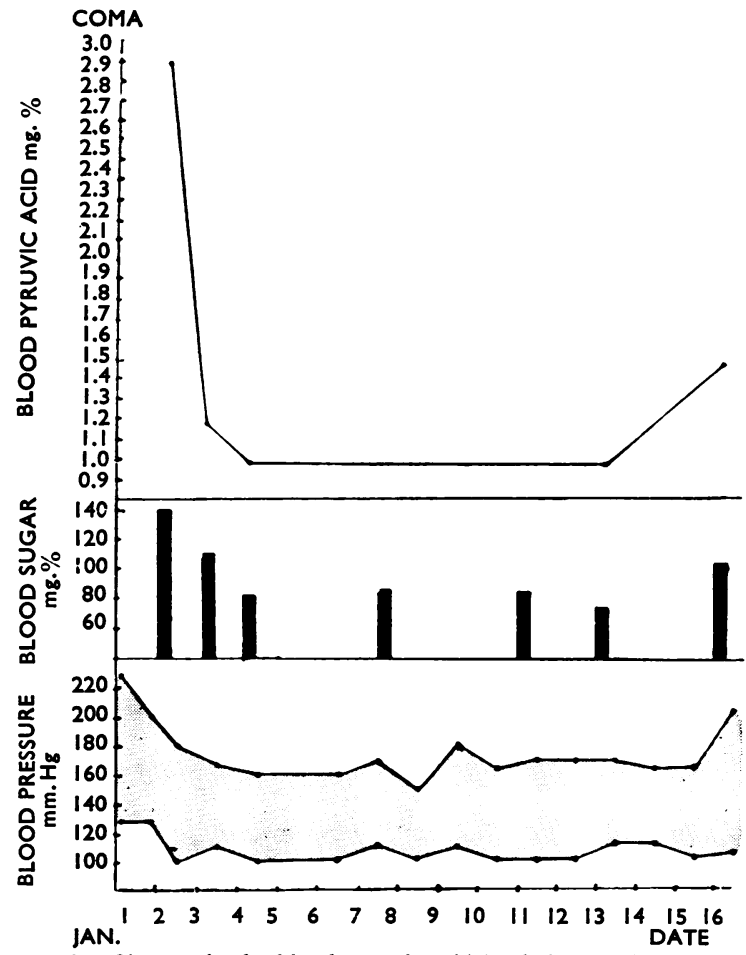

FIG. 2.-Changes in the blood pyruvic acid levels in a patient after a short episode of hypertensive encephalopathy (Case 28). Note the parallel blood sugar values. 


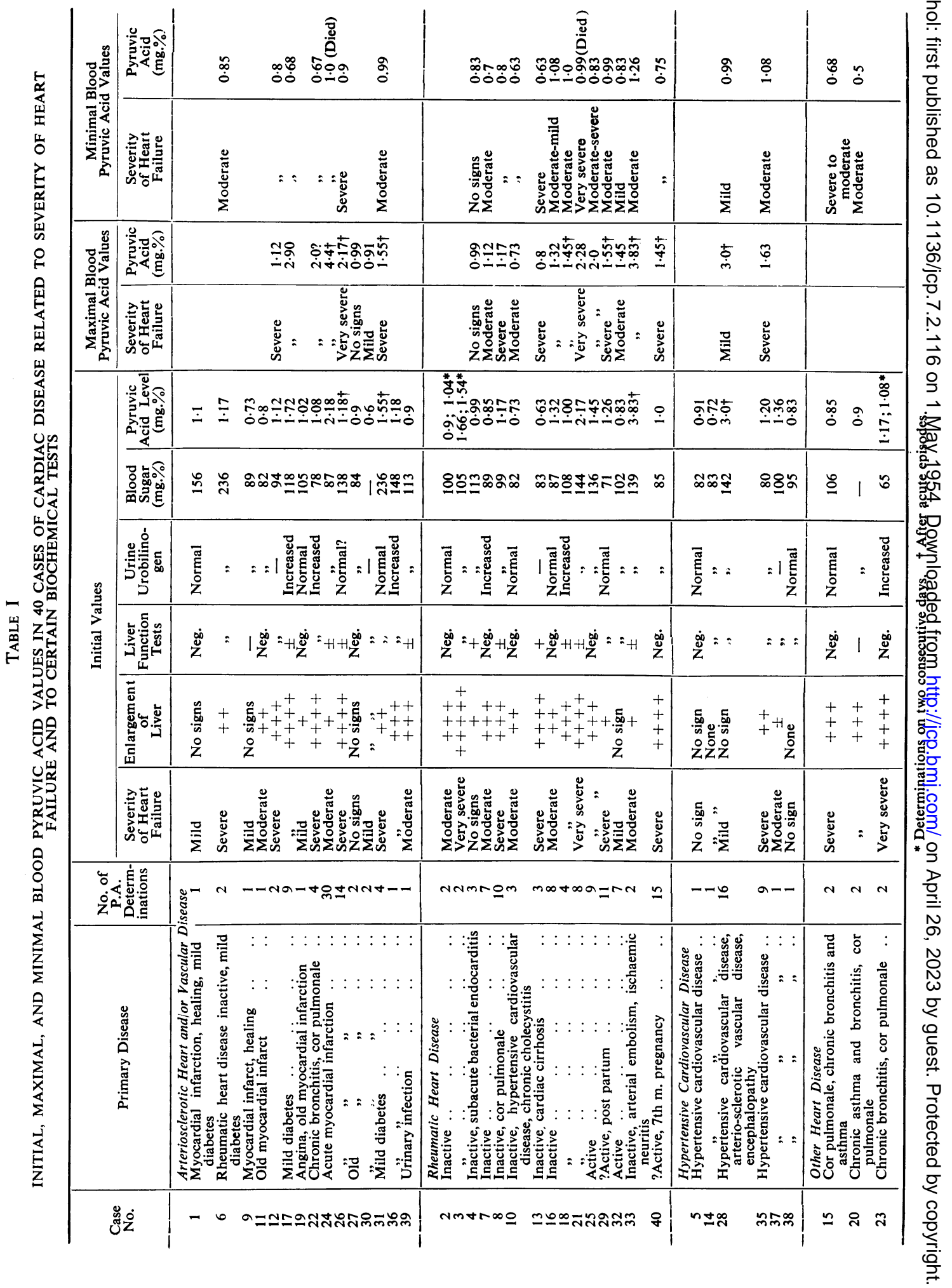


myocardial infarction, with a new rise of blood pyruvic acid to a very high level several hours before the clinical signs of the new attack. Co-carboxylase decreased the pyruvic acid level for a short time without signs of clinical improvement.

Case 28 (Fig. 2).-B. S., a man aged 46, had hypertensive cardiovascular disease, an old myocardial infarction, and residual hemianopia following a recent cerebral event. He was in mild heart failure. The blood pressure was usually $180-200 / 100-110$ mm. Hg.

He was admitted to hospital on January 1, 1952 , in a state of unconsciousness which had developed suddenly after a rise in blood pressure to $225 / 150 \mathrm{~mm}$. $\mathrm{Hg}$. He recovered consciousness within a few hours of admission. He was exhausted and vomiting, but no pathological change was seen in the neurological or other clinical findings other than the old hemianopia. Next morning the patient had a high fasting blood pyruvic acid level, and the blood sugar level was elevated with transient glycosuria. Liver tests were negative. Rapid clinical improvement on the first day was maintained during the following days, the pyruvic acid and sugar dropping to normal; the blood pressure was moderately raised. On January 16 he was again "feeling bad," and the blood pressure and blood pyruvic acid and sugar levels had all risen. No change in the degree of heart failure was discernible. Within one more day recovery was complete.

\section{Discussion}

Technique.-The hydrazin method of $\mathrm{Lu}$ and of Friedemann-Haugen has been challenged by several authors (Elgart and Nelson, 1941 ; Klein, 1941 ; Lestradet and Guest, 1951), as it is an estimation of keto-acids in general and not only of pyruvic acid. Markees (1951b) has shown that boiling the filtrate for five minutes destroys the aceto-acetic acid (the main competitor of pyruvic acid) without loss of pyruvic acid.

Normal Values.-The normal values for blood pyruvic acid in healthy persons obtained in this study $(0.53-1.08 \mathrm{mg} . \%)$ are in conformity with other reports irrespective of the methods used (Bueding, Wortis, and Stern, 1942 ; Friedemann and Haugen, 1943 ; Lu, 1939; Markees, Käser, and Lanz, 1950).

Relation between Degree of Failure and Pyruvic Acid Level.-Fig. 1 shows clearly that patients with heart disease, but without any clinical signs of failure, and patients with only mild symptoms of failure have normal blood pyruvic acid values. This fact is to be expected and it is likewise conceivable that very severe failure could raise the blood pyruvic acid as a sign of disturbed metabolism. On the other hand, several moderately severely and even very severely ill patients showed throughout the weeks of observation an almost normal blood pyruvic acid level. Both results are in agreement with the reports of other authors (Barenghi et al., 1941 ; Torre et al., 1950 ; Yanof, 1942). Even a very high blood pyruvic acid value, however, does not necessarily mean a fatal outcome, as was shown in two patients with a pyruvic acid level of 3 and of $4 \mathrm{mg}$.\%. Their recovery was accompanied by a return of the pyruvic acid level to almost normal figures.

When the pyruvic acid determinations revealed nearly normal figures despite the moderate or severe state of failure an improvement of the patient's condition sometimes decreased slightiy the values from 1.0 or 0.9 to 0.8 or 0.7 . Improvement of failure is generally followed by a drop in abnormally high blood pyruvic acid values, whereas a deterioration mostly caused a rise in the pyruvic acid level. As a rule, sudden complications elevated the blood pyruvic acid higher and more rapidly than a slow deterioration in a prolonged chronic illness.

It is of interest that in cases No. 8, 28, 29, 34, and 40 the elevation of the pyruvic acid level preceded by several hours and even for a day or two the appearance of clinical signs of deterioration. Should this finding be confirmed in a greater number of cases, it might not only prove valuable as a prognostic sign, but it might also demand from the physician greater vigilance when it occurs in a chronically ill patient with apparently innocuous complaints.

\section{Relation between Liver Function Tests and Blood Pyruvic Acid Level}

Severe liver damage may cause a rise in the blood pyruvic acid level (Davis and Bauer, 1944 ; Marche and Marnay, 1946; Amatuzio and Nesbitt, 1950 ; Kleeberg and Gitelson, 1953) because the damaged organ cannot metabolize this acid. On the other hand, severe heart failure is not infrequently associated with liver damage as a result of prolonged stasis and anoxia (Lichtman, 1949). It is therefore conceivable that liver insufficiency may be responsible, at least partly, for the rise in pyruvic acid level in severe heart failure (Amatuzio and Nesbitt, 1950).

In several of the severely ill patients a marked rise in pyruvic acid level coincided with deterioration in liver function (not indicated in Table I). However, in general there was no close correlation between the changes in pyruvic acid level and in the results of the liver tests employed. This may be partly explained by the fact that these tests do not represent all facets of the manifold functions of the liver (King, 1952 ; Popper, 1952 ; Staub, 1950). 
Influence of Therapy.-Since only the severe or very severe cases of failure had an elevated blood pyruvic acid level the entire well-established therapeutic regime had to be instituted without taking the risk of evaluating one procedure alone. Thus we can only state that general therapy lowers high pyruvic acid values if the clinical picture improves. On the other hand, if the severity of the circulatory failure persists, with or without treatment, the high pyruvic acid figures remain unaltered.

In 13 patients an additional therapeutic trial was made with a phosphorylated thiaminchloride (cocarboxylase) intravenously.* Williams, Egana, Robinson, Asper, and Dutoit (1942) reported that intravenous injection of di-phospho-thiamine suppressed the high pyruvic acid level in thyrotoxic patients. In a series of animal experiments, as well as in clinical studies on diabetics and on pregnant women, Markees has urged the therapeutic use of co-carboxylase. His results (Markees and Meyer, 1949) were remarkable in alloxan-diabetic rabbits as well as in human beings with severe diabetes. The effect was especially striking in regulating acidosis.

Using this method, Torre et al. (1950) observed in cardiac patients with an elevated pyruvic acid level a drop of almost $29 \%$ after the intravenous administration of co-carboxylase, whereas the injection of $100 \mathrm{mg}$. vitamin $B_{1}$ alone depressed the level by only $6 \%$. Lasch (1952) noted a significant therapeutic advantage with this phosphorylated thiamin in children with toxic diphtheria, who, of course, at the same time received specific therapy.

Our experiences with this substance are that in a few cases with an initially high blood pyruvic acid level the levels were reduced one or two hours after a single intravenous injection of 0.05 cocarboxylase. In some other patients with a pyruvic acid level at the upper border of normal, no such effect was seen. In three severely ill patients with an initially high blood pyruvic acid level a daily administration of 0.05 or 0.1 co-carboxylase caused a prompt decrease to nearly normal figures, associated with a mild clinical improvement. In two other patients, clinical improvement and a drop in the blood pyruvic avid level were obvious after the co-carboxylase therapy. One extremely ill patient died, however, in spite of a return to normal of the blood pyruvic acid level. In five cases after a single injection of 0.05 co-carboxylase or after repeated injections on alternate days no unequivocal effect on the blood pyruvic acid level

\footnotetext{
* Supplied through the courtesy of Hoffmann-LaRoche, Basle.
}

was observed when the blood was examined 24 hours after the injection.

Probable Mechanism of Pyruvic Acid Changes? in Congestive Heart Failure.-There is no facile explanation for fluctuations in the blood pyruviç acid level in cases of congestive heart failureฉ The most plausible explanation would be that anoxaemia of tissues causes this intermediate meta $\overrightarrow{0}$ bolic substance to accumulate (Ochoa, 1939 ; Torre et al., 1950 ; Yanof, 1942).

It is well known that anoxia increases the break down of glycogen and decreases the oxidation of: its split-products, pyruvic acid and lactic acid, in the liver. This leads to a rise in the concentration of these products, as well as of glucose, in the blood.

Actually several of the patients with severe heart? failure or myocardial infarction had simultane- 3 ously high blood pyruvic acid and high suga values.

However, our studies, as well as those by others, show that many patients with moderate or even more severe signs of heart failure still have ano almost normal blood pyruvic acid level. This wass particularly evident in patients with chronic coro pulmonale and long-standing failure. Rasmusseno and Storstein (1951) found almost normal arteriak oxygen saturation in many patients with chronic heart failure and dark-blue cyanosis. It is there- 3 fore possible that some of the chronically ille patients, even those in severe failure, did not have marked anoxaemia. On the other hand, Amatuzio and Nesbitt (1950) found in patients with pulmonary emphysema and marked hypoxia almosts normal pyruvic acid values. This seems to indi cate that hypoxia by itself, unless very severe, is not sufficient to cause hyperpyruvicaemia.

As another explanation of hyperpyruvicaemia after myocardial infarction Altschule and Rosen $\frac{7}{5}$ feld (1947) point to the possibility of increased: catabolism as part of a general stress reaction (Selye, 1950).

\section{Summary and Conclusions}

Forty patients with congestive heart failure of various grades were examined for blood pyruvi\& acid by means of the Lu-Friedemann-Haugen? method. All patients were on bed rest and $i$ standard sodium-free diet supplemented with at least $50 \mathrm{mg}$. vitamin $B_{1}$ per day.

Our normal figures for blood pyruvic acid in $1 \frac{\text { f }}{\mathrm{D}}$ healthy individuals conformed with the values found by other authors, by means of the same of by other methods of determination, with values ranging from 0.53 to $1.08 \mathrm{mg}$. \% 
The majority of the severe and all very severe cases had high blood pyruvic acid values. Every deterioration, especially acute, was accompanied by a rise in pyruvic acid. Improvement of the clinical picture was paralleled by a decrease of blood pyruvic acid, often to normal figures. Even high values, 3 or $4 \mathrm{mg}$. $\%$ need not necessarily indicate a fatal outcome.

One blood pyruvic acid determination in cases of congestive heart failure is only of minor value because mildly or moderately ill patients and some severely ill patients showed normal or almost normal values; chronic cases may do so for a considerable length of time in spite of severe heart failure.

In several patients, in whom serial blood pyruvic acid determinations were carried out, an elevation of pyruvic acid to above normal preceded the appearance of clinical symptoms of deterioration or of complications. It would appear, therefore, that repeated pyruvic acid tests might be of prognostic value if associated with other clinical and biochemical findings.

There was no significant relation between the blood pyruvic acid level and the results of some of the so-called liver tests. A definite connexion could be evaluated in certain cases of heart failure between blood sugar and pyruvic acid values.
In 13 cases thiaminpyrophosphate was administered intravenously to patients who at the same time received the usual cardiac therapy. In cases receiving repeated injections the elevated blood pyruvic acid dropped to nearly normal, but was not uniformly accompanied by marked clinical improvement.

\section{REFERENCES}

Altschule, M. D., and Rosenfeld, F. M. (1947). Arch. intern. Med.,

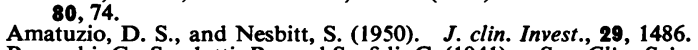

Barenghi, G., Scarlotti, R., and Scafidi, C. (1941). Sec. Clin. Scient., 5.

Bueding, E., Wortis, H., and Stern, M. (1942). J. clin. Invest., 21, 85.

Davis, H. A., and Bauer, F. K. (1944). Arch. Surg., Chicago, 48, 190.

Elgart, S., and Nelson, N. (1941). J. biol. Chem., 138, 443.

Friedemann, T. E., and Haugen, G. E. (1943). Ibid., 147, 415.

King, W. E. (1952). In Modern Trends in Gastro-enterology, ed. Jones, F. Avery, pp. 671-691. Butterworth, London.

Kleeberg, J., and Gitelson, S. (1953). Harefuah, 45, 152.

Klein, D. (1941). J. biol. Chem., 137, 311.

Lasch, F. (1952). Ann. paediat., Basel, 178, 333.

Lestradet, H., and Guest, G. M. (1951). Presse méd., 59, 375.

Lichtman, S. S. (1949). Diseases of the Liver, Gallbladder and Bile Ducts, 2nd ed. Lea and Febiger, Philadelphia.

Lu, G. D. (1939). Biochem. J., 33, 249.

Marche, J., and Marnay, C. (1946). C.R. Soc. Biol., Paris, 140, 785.

Markees, S. (1951a). Schweiz. med. Wschr., 81, 1145.

(1951b). Experentia, Basel, 7, 314.

- and Meyer, F. W. (1949). Schweiz. med. Wschr., 79, 931.

Käser, O., and Lanz, R. (1950). Ibid., 80, 1079.

Ochoa, L. (1939). Biochem. J., 33, 1262.

Popper, H. (1952). Rev. Gastroent., 19, 826.

Rasmusen, H., and Storstein, O. (1951). Acta med. scand., 141, 43.

Selye, H. (1950). Stress. Acta, Montreal, Canada.

Staub, H. (1950). Helv. med. Acta, 17, 376.

Torre, L. I., Teece, G., and Baldantoni, A. (1950). Quad. Nutr., 11, 1.

Williams, R. H., Egana, G., Robinson, P., Asper, S. P., and Dutoit, C. H. (1942). J. clin. Invest., 21, 623 .

Yanof, Z. A. (1942). Arch. intern. Med., 69, 1005. 